\title{
Flight cost calculation for unmanned air vehicle based on path length and heading angle change
}

\author{
Sanjoy Kumar Debnath ${ }^{1}$, Rosli Omar ${ }^{2}$, B. S. S. K. Ibrahim ${ }^{3,}$ Susama Bagchi $^{4}$, Elia Nadira ${ }^{5}$, \\ Faisal Amin', Bashir Bala Muhammad ${ }^{7}$ \\ 1,2,3,4,5,6 Faculty of Electrical \& Electronics Engineering, Universiti Tun Hussein Onn Malaysia, Malaysia \\ ${ }^{3}$ School of Mechanical, Aerospace \& Automotive Engineering, Faculty of Engineering, Environment \& Computing, \\ Coventry University, United Kingdom \\ ${ }^{7}$ School of Mechanical Engineering, Northwestern Polytechnical University, China
}

\section{Article Info \\ Article history: \\ Received Aug 12, 2019 \\ Revised Oct 28, 2019 \\ Accepted Dec 3, 2019}

\section{Keywords:}

Algorithm development

Autonomous

Cost calculation

Energy efficient

UAV path planning

\begin{abstract}
This paper proposes a method to calculate a flight cost of an unmanned aerial vehicle (UAV) considering its change of heading angle though there are many reasons that cause the energy consumption. The proposed approach demonstrates that when a UAV moves from a starting position/point to a target/goal position/point, if the number of obstacle increases, the number of heading change would also increase. As a result, it raises the energy consumption of the UAV. It also shows that the magnitude of heading change would affect the energy consumption proportionally. The theoretical analysis as well as the simulation outcome proves the usefulness of the proposed technique.
\end{abstract}

This is an open access article under the CC BY-SA license.

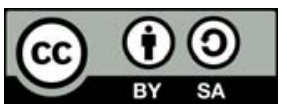

\section{Corresponding Author:}

Rosli Omar,

Faculty of Electrical \& Electronic Engineering,

Universiti Tun Hussein Onn Malaysia,

Parit Raja, Batu Pahat-86400, Johor, Malaysia.

Email: roslio@uthm.edu.my

\section{INTRODUCTION}

A path planning assignment usually takes several input values or parameters; such as a starting position/point or start configuration which is an initial and major configuration of UAVs, a goal position/point or goal configuration which is the basic configuration of a UAV, and the obstacles which are the objects to be avoided by the UAV while traversing a path from the starting configuration/point to the goal configurations/point. A path, relating to the starting configuration and the goal configuration, can be specified in a configuration space. Moreover, extra constraints typically make the mission progressively complicated like keeping a specific distance threshold to the obstacles or moving in only one direction etc. A path-planning algorithm for a moving UAV, by its own strategies, must avoid the obstacles to maintain safety.

A UAV has a vast usability. Their agility promotes them to be engaged in numerous surveillance and public related works, such as, localities [1] and wildlife observing [2], search as well as rescue operations [3], and also in cultivation [4]. NASA first time built and tested unmanned aeronautical framework for its safe and competent low-altitude airspace traffic management [5].

J. Giesbrecht and Defense R\&D Canada used a synopsis of high-level path planning methods and surveyed all bits of the path planning procedure as well as world representation, graph search algorithms, and 
planning for partly and entirely indefinite environments [6]. Planning representations such as Roadmaps Technique (RM), Cell Decompositions (CD) and Potential Fields (PF) alongside heuristic as well as nonheuristic methods of graph search were covered. Recently, advanced and widespread algorithms were also examined such as $A^{*}$ then $D^{*}$, Potential Fields (PF), Rapidly Exploring Random Trees (RRT) and Probabilistic Roadmaps (PRM) [6, 7]. Classic methods have numerous shortcomings, like high time complexity with high dimensions, and occurrence of local minima, and hence, these algorithms are incompetent. So, to make the classic methods proficient, probabilistic algorithms were used with PRM and RRT to reap the foremost advantage of high-speed implementation. To solve the problem of local minima, many heuristics as well as meta-heuristic algorithms were used in robot motion planning. For instance, a combination of the PF and Simulated Annealing (SA) technique was the remedy of this problem. Further approaches were Genetic Algorithms (GA), Artificial Neural Network (ANN), Particle Swarm Optimization (PSO), Wavelet Theory, Ant Colony (ACO) and Fuzzy Logic (FL). Heuristic algorithms never give assurance to find a solution, but if they (algorithms) do, it is expected to be more rapid than the deterministic approaches [8].

\section{ENERGY EFFICIENT PATH PLANNING ISSUES}

Energy efficient path planning is a complex subject. Many entities need to be measured for calculating the energy efficient path planning. Some of them stated below are usually considered in the energy cost calculation for optimal energy efficient path planning concern:

\section{i. Path Distance}

Completion of a mission depends on the arrival of a UAV to a target point after going through the planned waypoints. Thus, the core importance is given to path distance that is the travelled distance between the starting and the target point.

\section{ii. Path Travel Time}

Travel time is also another measure of optimal energy efficient path planning algorithm. For this instance, it considers that the quickest and the shortest paths are different. The quickest/fastest means the vehicle can reach its target within the least travel period. The quickest path may not be the same as the shortest path because of the traffic and haphazard incidences just as the flying or driving guidelines like limited range of speed. Additionally, the fastest path must be updated frequently throughout the vehicle trip as traffic circumstances change quickly, particularly in huge urban areas.

\section{iii. Path Heading Change}

If UAV travels in a straight path without any obstacle, its speed may be constant. However, if there is obstacle on the flight path, the UAV needs to take a suitable alternative route avoiding the obstacle by changing its direction starting from the nearest waypoint. Therefore, its speed has to be slowed down while passing the waypoint to ensure it is collision-free. The UAV may need to accelerate again after avoiding the obstacle to ensure that the mission is performed within the given time. Every time the UAV changes its speed, it will cause energy loss and hence, with the increasing number of obstacles, the loss will increase proportionally.

\section{iv. Safety}

Most optimal energy efficient path planning algorithms emphasis on the shortest path finding when other qualities of service also deserve attention such as distance, obstacles, physical limitations of the vehicle, algorithm plan, environment, optimality, completeness, space and time complexity, dynamics etc. Among them, safety is always the first priority in a UAV mission.

\section{v. Obstacle Hostility}

Obstacles' hostility is the ratio between the area blocked by obstacles in a given free space and the size of the total free space area. To find the available path that the UAV can follow, the description of the configuration space is required [9]. Configuration space (C-space) is the common idea behind almost all path planning approaches and it mainly consists of three elements namely workspace, free space and obstacle [10].The UAV must have prior knowledge about the obstacle area and the free space so that it can find its path from starting to target point ensuring no collision. Considering this matter, configuration space is the area where the UAV can fly by avoiding collision and find the shortest path and, as a result, it can save energy. Hence, the configuration space indicates the actual obstacle zone and free space region for the travers

Flight cost calculation for unmanned air vehicle based on path length and heading (Sanjoy Kumar Debnath) 
of UAV. If the total search area is A and the total area blocked by obstacles is $\mathbf{O}$ then the obstacles hostility $=\mathbf{O} / \mathbf{A}$.

\section{vi. Weather}

In open-air flight, UAVs distinctly need to deal with the stochastic of weather circumstances which can impact the energy feeding of UAVs [11-13]. These ensure several characteristics that can potentially and powerfully influence the solution approach routing problem for a UAV. There are two key issues of weather's influences on the UAV moving and they are described below.

Wind: Wind is the foremost environmental influence that disturbs the UAV because of its direction of flow and speed. Wind may give benefit to the energy consumptions or give bigger resistance to the movement in other scenarios [14].

Temperature: The sceneries of temperature is able to disturb the UAV's battery provision as it is interrelated to drain battery and its capability [15].

vii. UAV Flying Speed and Payload

The relative and rational flying speediness of the UAV is a precarious issue to determine the fuel consumption. Direction of the Wind speed is related with the flying speed because wind direction disturbs the flying standard of the UAV, either positively or negatively. The flying position of a UAV can situate at any subsequent: a. hovering and b. level flight, cruising or horizontal moving also c. vertical moving: vertical take-off/ altitude adjustment /landing alteration. Therefore, the flying condition of the UAV must be measured along with the flying speediness in computing the energy feeding [14]. Besides that, the related models are proposed in section 3 with relative en route for these flight statuses.

Normally, UAVs carry specific forms of payloads, for instance, camera kit or parcels. Effect of the dissimilar masses of payloads might be significant when deriving the model of energy consumption [14, 15]. In aircraft engineering, it is recognized that the energy/fuel consumption is subject to certain factors. Perhaps, maximum flight time or flight distance of UAV may be constrained by takeoff total weight, overweight, empty weight and thrust to the weight ratio [16], payload, and fuel weight [17]. Since the UAV's engineering/manufacturing, individual can get equivalent prototypes intended for flight for example, existing/obtainable fuel replicas for multi-rotor helicopters [18] which demonstrate the linear estimation of the energy ingesting is not appropriate aimed at huge deviations of the payload conveyed [15].

viii. Computational complexity

This is a metric associated with computational performance of an algorithm. It is important that the computational complexity of every algorithm needs to be considered, because computational time causes energy consumption [19].

ix. Scalability:

The assessment of a path planning algorithm for an autonomous vehicle or UAV is considered as scalability. Scalability is a state when with a larger network, the performance of an algorithm declines. Hence, a well performed algorithm which is designed for trivial path network probably won't be appropriate for bigger path networks.

x. Quality of the best path:

This metric is utilized to compare the multiple finest paths that are planned and computed by altered heuristics and supportive to similar metrics (i.e. travel time, travel distance etc.) with the aim of deciding which algorithm is manipulative in obtaining the nearest answer/clarification to the ideal or optimal path.

\section{FLIGHT COST CALCULATION METHOD AND ANALYSIS}

Consider a UAV's path as depicted in Figure 1. The path starts at $\boldsymbol{S}$ and ends at $\boldsymbol{G}$ through a waypoint $\boldsymbol{W}$. Note that, in the figure, $\Theta=\angle S W G$ is $180^{\circ}$ which means no UAV's heading change throughout the path. Therefore, the UAV traverses the path in a straight line from point $S$ to $G$.

The cost, $D_{\theta}$ can be calculated using the following (1)

$$
\mathrm{D}_{\theta}=\mathrm{D}\left(1+\sin \left(180^{\circ}-\theta^{\circ}\right)\right)
$$

Int J Pow Elec \& Dri Syst Vol. 11, No. 1, Mar 2020 : 382 - 389 


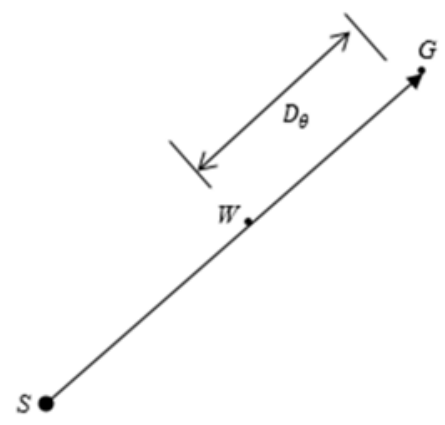

Figure 1.UAV path from starting point $S$ to goal point $G$ through a waypoint $W$ with no heading change

The equation implies that, if there is no heading angle change on the path towards the goal, then the total cost is only the distance of the path.

Figure 2 depicts a path, which starts at $S$ and ends at goals $G_{l}$ to $G_{10}$. Assume that the UAV has to traverse the path and after reaching at the way point $W$, it changes its heading angle towards goal points $G_{1}$ to $G_{10}$ with the angles of $\theta=180^{\circ}, 170^{\circ}, 160^{\circ} 150^{\circ}, 140,130^{\circ}, 120^{\circ}, 110^{\circ}, 100^{\circ}$ and $90^{\circ}$ respectively.

It can be interpreted that for an angle of $170^{\circ}$, the UAV has to make a $10^{\circ}$ heading change. This results in the minimum UAV speed change to cater the turn. After passing $W$, the UAV's speed will be restored to the normal one. The slight change in the UAV speed (slows down and speeds up) will result in small energy loss. For the worst-case scenario, where the heading change is $90^{\circ}$, the UAV has to instantly reduce its speed and again rapidly increase its speed to the normal one after passing $W$. This causes the UAV to consume considerably more energy compared to the former scenario.

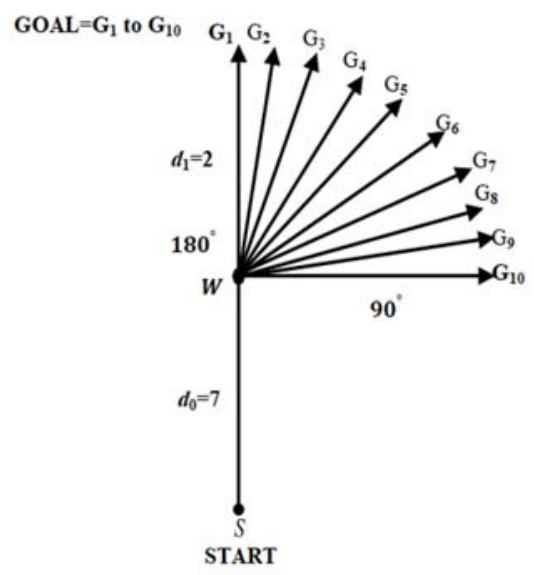

Figure 2. UAV path with different goal considering different angle

Therefore, from (1) and referring to Figure 2, can be constructed as (2)

$$
\text { Cost }=d_{0}+d_{1 \theta}=d_{0}+d_{1}\left(1+\sin \left(180^{\circ}-\theta\right)\right)
$$

The calculated costs from $S$ to $G_{i}$ be listed in Table 1 considering Figure 2 and assuming that the distance between $S$ and $W$ is $7 \mathrm{~m}$ and $W$ to $G_{i}$ is $2 \mathrm{~m}$.

In Table 1, it is clearly observed that the gradual increment in the angles causes the gradual raise in energy consumption.

Flight cost calculation for unmanned air vehicle based on path length and heading (Sanjoy Kumar Debnath) 


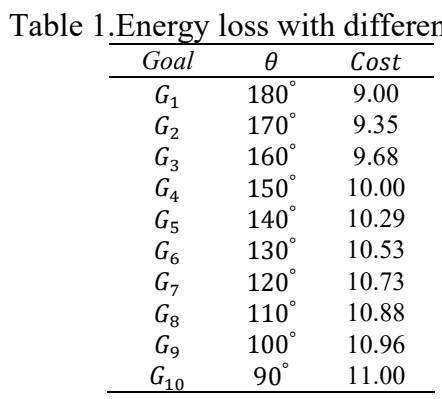

\section{SIMULATION RESULT AND DISCUSSION}

The proposed equation in the previous section is applied to find the cost of a randomly generated path shown in Figure 3. The UAV has to traverse the path from the starting point $S$ to goal point $G$ with multiple heading changes. Here, the obstacles are not shown.

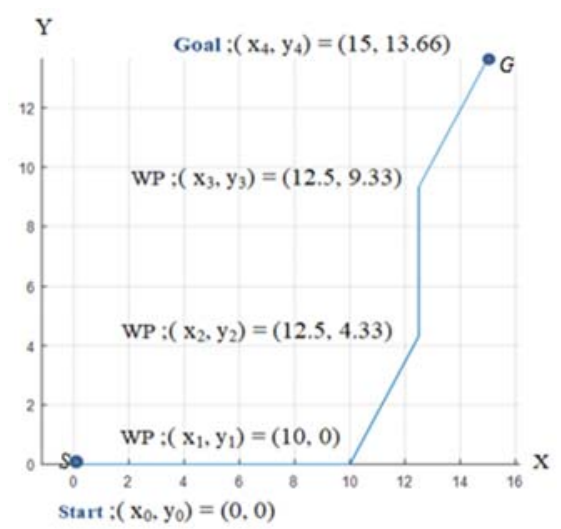

Figure 3. The UAV has to traverse the path from $S$ to $G$ point with multiple heading changes

The total length of the path without considering the heading changes is $25 \mathrm{~m}$. But, once the changes in the heading angle are taken into account then total cost is $34.33 \mathrm{~m}$.

Generally, multiple heading angle changes occur due to the increase in the number of turns with the increasing number of obstacles in a UAV path. In a straight path, UAV maintains a steady speed. In a path with piece-wise linear segments which are not parallel, the UAV has to change its heading angle near the waypoints to avoid obstacles. Changing the heading angle will result in reduced speed (deceleration). After passing the waypoint, the UAV may start to accelerate again. This deceleration and acceleration will lead to more energy consumption [20].

Furthermore, Table 1 clearly demonstrates that the greater heading change causes additional energy consumption and increases the path cost. Figure 3 also illustrates the same theory of more energy consumption with increasing heading angle change of a UAV.

\section{FUTURE WORK}

In $[9,21]$, it is shown that for optimal energy efficient path planning for an autonomous vehicle or unmanned air vehicle, particularly two algorithms are best fit. They are visibility graph (VG) under combinatorial method and Dijkstra's algorithm under graph search algorithm. Both methods are very good in terms of optimization and completeness quality. If we apply both the algorithms for more energy saving, it is possible to suggest a range of angles that have low energy consumption cost. For example, from Table 1, angles of $\angle 180^{\circ}-\angle 130^{\circ}$ has a low energy consumption.

The main reasons for UAVs to be more popular these days in providing technical assistance is because of its autonomous use in adverse situations to accomplish hazardous tasks without any involvement

Int J Pow Elec \& Dri Syst Vol. 11, No. 1, Mar 2020 : $382-389$ 
of human beings. The key enabler in improving an autonomous industry is the "decentralized decision" for which a UAV can autonomously travel a pre-defined path in between the starting point and target point. An UAV can communicate through the Internet of Things (IoT) due to the "Interoperability" which can be considered in the advance stages of a project for "Information transparency". In addition, the desired outcome can also be obtained with the selected algorithm by implementing the essential key technologies for Industry 4.0 transformation such as adaptive robotics partly or fully, cloud systems, cyber security, machine learning, artificial intelligence, and IoT [22-25].

\section{CONCLUSION}

This study introduced a new technique to calculate the flight cost of an unmanned air vehicle considering its heading angle changes for an optimal energy efficient path planning approach. Besides that, some other factors are also discussed which influenced the energy consumption. The new method examined the general characteristics of the energy ingesting or consumption for a UAV's path planning. The result revealed that the energy consumption of a UAV increased due to the increasing number of heading angles as a result of the growing number of obstacles. The theoretical and mathematical analysis and outcomes via simulation proved the efficacy of this method. The significance of the stealthy path is immense in terms of saving energy in UAV path planning.

\section{ACKNOWLEDGEMENTS}

The authors would like to give special thanks to Ministry of Higher Education Malaysia and Universiti Tun Hussein Onn Malaysia associate with Research Management Center, for the research fund support under TIER-1 VOT H131.

\section{REFERENCES}

[1] Yang L, Qi J, Song D, Xiao J., Han J., Xia Y., "Survey of robot 3D path planning algorithms," Journal of Control Science and Engineering, Vol. 1, No. 5, Mar 2016

[2] Waharte, Sonia, and Niki Trigoni. "Supporting search and rescue operations with UAVs," Emerging Security Technologies (EST), 2010 International Conference on. IEEE, 2010.

[3] Tripicchio, Paolo, Massimo Satler, Giacomo Dabisias, Emanuele Ruffaldi, and Carlo Alberto Avizzano. "Towards smart farming and sustainable agriculture with drones." In Intelligent Environments (IE), 2015 International Conference on, IEEE, pp. 140-143, 2015.

[4] "UAS traffic management," [Online] Available http://www.utm.arc.nasa.gov/utm2015. shtml.Engineering 2016

[5] Ahmed, Shaimaa, Amr Mohamed, Khaled Harras, Mohamed Kholief, and Saleh Mesbah. "Energy efficient path planning techniques for UAV-based systems with space discretization." In Wireless Communications and Networking Conference (WCNC), 2016 IEEE, pp. 1-6. IEEE, 2016.

[6] Giesbrecht, J. Global path planning for unmanned ground vehicles. No. DRDC-TM-2004-272, "Defence research and development suffield (alberta)," 2004, M. Young, The Technical Writer's Handbook. Mill Valley, CA: University Science, 1989.

[7] LaValle, Steven M., "Planning algorithms," Cambridge university press, 2006.

[8] Masehian, Ellips, and Davoud Sedighizadeh. "Classic and heuristic approaches in robot motion planning-a chronological review," World Academy of Science, Engineering and Technology, vol. 29, No. 1, pp.101-106, 2007.

[9] Debnath, Sanjoy Kumar, Rosli Omar, and Nor Badariyah Abdul Latip. "A review on energy efficient path planning algorithms for unmanned air vehicles," In Computational Science and Technology, pp. 523-532. Springer, Singapore, 2019.

[10] Omar, Rosli bin, "Path planning for unmanned aerial vehicles using visibility line-based methods," PhD diss., University of Leicester, 2012

[11] Yu, V.F., Lin, S.-W., "Solving the location-routing problem with simultaneous pickup and delivery by simulated annealing," Int. J. Prod. Res, vol 54, pp. 1-24, 2015.

[12] Qian, Z., Wang, J., Wang, G., "Route planning of UAV based on improved ant colony algorithm," pp. 1421-1426, 2015

[13] Sarıçiçek, İ., Akkuş, Y., "Unmanned aerial vehicle hub-location and routing for monitoring geographic borders," Appl. Math. Model, vol. 39, pp. 3939-3953, 2015.

[14] Tseng, C-.M., Chau, C-.K., Elbassioni, K., Khonji, M., "Autonomous recharging and flight mission planning for battery-operated autonomous drones, pp. 1-10, 2017.

[15] Dorling, K., Heinrichs, J., Messier, G. G., Magierowski, S.,"Vehicle routing problems for drone delivery," IEEE Trans. Syst. Man Cybern. Syst., vol. 47, pp. 1-16, 2016.

[16] Shetty, V.K., Sudit, M., Nagi, R.,"Priority-based assignment and routing of a fleet of unmanned combat aerial vehicles," Comput. Oper. Res., vol. 35, pp. 1813-1828, 2008.

[17] Zhang, J., Jia, L., Niu, S., et al., "A space-time network-based modeling framework for dynamic unmanned aerial vehicle routing in traffic incident monitoring applications," Sensors (Switzerland), vol. 15, pp. 13874-13898, 2015.

Flight cost calculation for unmanned air vehicle based on path length and heading (Sanjoy Kumar Debnath) 
[18] Leishman, D. S., (Eng. PDFRASJG) Principles of Helicopter Aerodynamics, 2006.

[19] Latip, Nor Badariyah Abdul, Rosli Omar, and Sanjoy Kumar Debnath, "Optimal path planning using equilateral spaces oriented visibility graph method," International Journal of Electrical and Computer Engineering (IJECE), vol. 7, No. 6, pp. 3046-3051, 2017.

[20] Binitha, S., and S. Siva Sathya, "A survey of bio inspired optimization algorithms," International journal of soft computing and engineering, vol. 2, No. 2, pp. 137-151, 2012.

[21] Debnath, Sanjoy Kumar, Rosli Omar, and Nor Badariyah Abdul Latip. "Comparison of different configuration space representations for path planning under combinatorial method," Indonesian Journal of Electrical Engineering and Computer Science, vol. 1, No. 1, pp. 401-408, 2019.

[22] Hermann M, Pentek T, Otto B., "Design principles for industrie 4.0 scenarios," In System Sciences (HICSS), 49th Hawaii International Conference. IEEE, pp. 3928-3937, 2016.

[23] Sarvari PA, Ustundag A, Cevikcan E, Kaya I, Cebi S., "Technology roadmap for industry 4.0: Managing The Digital Transformation," Springer, Cham, pp. 95-103, 2018.

[24] Santucci, Gérald. "The internet of things: Between the revolution of the internet and the metamorphosis of objects," Vision and Challenges for Realising the Internet of Things, pp. 11-24, 2010.

[25] Mattern, Friedemann, and Christian Floerkemeier, "From the internet of computers to the internet of things," From active data management to event-based systems and more, Springer, Berlin, Heidelberg, pp. 242-259, 2010.

\section{BIOGRAPHIES OF AUTHORS}
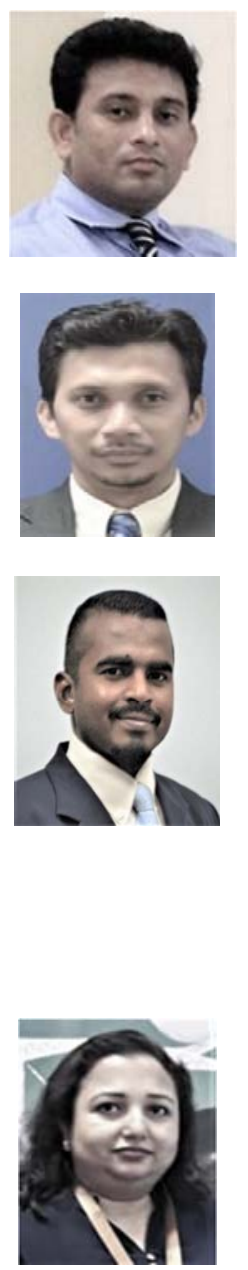

Sanjoy Kumar Debnath is a PhD scholar in Faculty of Electrical \& Electronic Engineering in the Universiti Tun Hussein Onn Malaysia (UTHM). He received his Masters of Engineering from Universiti Teknologi Malaysia in 2014. He joined a research on "Optimal Energy Efficient Path Planning for an Unmanned Air Vehicle (UAV) in Obstacle-Rich Environment" in 2016 at UTHM under the Office of Research, Innovation, Commercialization, and Consultancy Management center.He is a "Graduate Engineer" from Board of Engineers Malaysia also a "Graduate Member" from Institute of Engineers Malaysia as well IEEE Malaysian section respectively.

Dr.Rosli Omar currently is an Associate Professor and Dean at the Faculty of Electrical and Electronic Engineering, Universiti Tun Hussein Onn Malaysia. He received his $\mathrm{PhD}$ in engineering from University of Leicester, United Kingdom in 2012. He is highly motivated academician with more than 15 years of teaching and research experience. His research interests are in robotic engineering, autonomous systems and system identification.

Dr. Babul Salam KSM Kader Ibrahim (PhD from Sheffield University, United Kingdom) is an Assistant Professor in Automation and Robotic of School of Mechanical, Aerospace and Automotive, Coventry University, United Kingdom. Prior to this appointment, he was an Associate professor in Department of Mechatronic and Robotic Engineering at University Tun Hussein Onn Malaysia, Johor, Malaysia. He is highly motivated academic with significant expertise in the field of mechatronics with special adherence to robotics and control systems with 10 years teaching experience combined with course work and research background. He has published more than 80 technical papers in journals and conference proceedings in these fields. Dr. Ibrahim is a Chartered Engineer of Institution of Engineering and Technology (IET) UK, Professional Engineer of Board of Engineers Malaysia and member of IEEE. His research interests include electric vehicle, mechatronics, nonlinear control and modelling, rehabilitation robotics and control in biomedical application.

Susama Bagchi obtained her MSc. in Modern Communication Technologies with Business Management from the University of Sussex, UK after completing the Bachelor of Engineering from the University of Burdwan, West Bengal, India. She is a Doctoral fellow at the Universiti Tun Hussein Onn Malaysia under the Faculty of Electrical and Electronic Engineering. She already gained 8 years of experience in corporate (engineering management) in different sectors. She is a "Graduate Engineer" from Board of Engineers Malaysia (BEM) and "Graduate Member" of Insitute of Electrical \& Electronics Engineers (IEEE). 


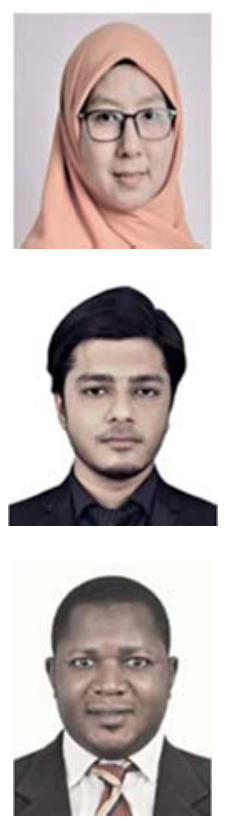

Elia Nadira Sabudin is a Doctoral fellow at the Universiti Tun Hussein Onn Malaysia under the Faculty of Electrical and Electronic Engineering. She received her Master of Engineering from Universiti Tun Hussein Onn Malaysia in 2012. She joined a research on "Efficient Cooperative path planning approach based on Potential field method using UAVs" in, 2013 at Universiti Tun Hussein Onn Malaysia.

Faisal Amin received his Bachelor of Electronics Engineering from Sir Syed University, Pakistan in 2015. He recieved his Masters of Electrical Engineering from Universiti Tun Hussein Onn Malaysia in 2019. His research interests include robotics, algorithm development, control system, image processing, power electronics, machine and drives.

Bashir Bala Muhammad received a B. Eng. in electrical from Bayero University Kano, Nigeria in 2010, and a Master of mechatronic engineering from Universiti Teknologi Malaysia, in 2015. He is currently pursuing his Ph.D. in mechatronic engineering at the School of Mechanical Engineering, Northwestern Polytechnical University, Xi'an, China. His current research interests in dynamic system identification, robotics, modeling, and control 